\title{
ACT-R Model for Addition Under Emotional Stimulus of Major Depressive Disorder and Control
}

\author{
Xiaojing Yang ${ }^{1,2^{*}}$, Yiting Wang ${ }^{1}$ \\ 1. College of Computer and information engineering, Anyang Normal University, Anyang 455000 \\ 2. International WIC Institute, Beijing University of Technology, Beijing 100124
}

\begin{abstract}
Neural correlate of major depressive disorder (MDD) and control of addition under emotional stimulus is still unclear. Addition under positive emotional stimulus (AP) and addition under negative emotional stimulus (AN) are two typical different cognitive processing, and with a common core component of rule induction. Previous studies have demonstrated that different processing is adopted in AP and AN completion tasks; even the underlying rules are identical. In the present study, we examined cortical activation as a function of two different machining processing for solving addition calculation under different emotional completion tasks. In AP completion tasks, involves direct retrieving of arithmetic knowledge to get the relations between items. In AN completion tasks, requires counting a certain number of times to detect the relations linking two items. The two processes require essentially the equivalent cognitive processes, but they have different working memory demands. In AN completion tasks produced significant greater activity involved in memory retrieval (dorsolateral prefrontal cortex, DLPFC) and mental representation/maintenance (posterior parietal cortex, PPC). An ACT-R model of the tasks successfully predicted the behavioral performance and BOLD response. The present findings positive emotional stimulus has promoting effect on addition cognitive calculate. Negative emotional stimulus has inhibiting effect on addition cognitive calculate.
\end{abstract}

\section{Introduction}

Major depressive disorder (MDD) ${ }^{[1-2]}$ is a significant and lasting low mood as the main mark, and with lower self-esteem, lack of pleasure and a series of symptoms of affective disorders ${ }^{[3]}$. It has the characteristics of chronic deferment, high incidence and easy recurrence, the suicide rate and high morbidity, work, study and daily life of the patients with many aspects, such as, interpersonal communication has a serious negative emotion ${ }^{[4]}$. Over the past 20 years, the development of neuroimaging provides a new train of thought to explore the neural mechanism of brain, which has become the indispensable important tool of disease physiological basis ${ }^{[5-6]}$. Through the comparative analysis of the normal people and MDD can be found, it may provide indicators for the diagnosis of the disease to reveal the development rule of disease, provide the basis for relevant treatment in the future. Researchers believe that these brain regions in the process of emotional and cognitive form regulation loop ${ }^{[7-9]}$. Researchers assumed that the structure and function of patients with depression anomalies mainly related to emotion recognition loop ${ }^{[10]}$. Depression of the frontal cortex, knee cortical volume decreases ${ }^{[11-12]}$. Task state fMRI experiments study found that patients with depression and emotional, cognitive processing part of the brain associated with memory function impairment ${ }^{[13-14]}$. Researchers found that MDD of the dorsolateral prefrontal cortex (dIPFC) has the abnormal activation of significant in cognitive processing, and were positively associated with the degree of cognitive load ${ }^{[15]}$. Relevant research shows that negative emotional stimulus has attention retention for cognitive task ${ }^{[16-18]}$.

Digital computing is one of the basic human brain cognitive functions ${ }^{[19]}$. Simple arithmetic natural mating, the simplest and most commonly used and has some related ${ }^{[20]}$. Dehaene and Riveral believes that a simple single digits addition and multiplication events (additive formula, my multiplication) in the process of calculating extraction depend on the participation of the basal ganglia ${ }^{[21]}$. Division operation and carry and abdication of addition and subtraction are complex, there have been many studies about digital cognition theory and experimental psychology, neuroimaging that a particular area of the brain with different digital processing function. The PFC and PPC are considered to be one of the most important areas ${ }^{[22]}$. This study is to choose a non-carry addition calculation equation under different emotional picture stimuli as the experimental paradigm, in order to studying the effect of different emotional stimuli to addition calculation, the positive, neutral, negative emotional stimuli pictures appear before an addition equation present. Experimental design is shown in figure 1, the participants only need to judge the two digits addition equation right and wrong under the emotional stimulus pictures if the result is right to press the left key, or else press right key.

ACT - R can simulate the behavior process and the characteristics of the brain functional areas in the process of physiological activity, can predict fine granularity time information processing (e.g., $0.2 \mathrm{~s}$ ) and the active model of ROI ${ }^{[23]}$. The shortcoming of fMRI technology is can't link brain activity with specific information processing, the shortage of the ACT-R simulation is unable to confirm the rationality of the forecast results,

\footnotetext{
Corresponding author: yangxj84@163.com
} 
ACT - R meets fMRI can be complementary advantages to avoid shortage. Zhong Ning et al. combined with artificial Intelligence, cognitive science and neuroscience research contents, advocate using BI (Brain Informatics) research to development of WI (Web Intelligence) applications ${ }^{[24]}$. John Anderson, Yinlin Qin et al study on Hanoi tower, solution of the equation, geometric proof et al. In their study, eight brain regions corresponding to different cognitive component, the parietal lobes activities reflect the state of problem space representation, left prefrontal activities reflect the declarative memory retrieval, basal ganglia activities reflect to extract the production rule, the area before the cingulate activities reflect the target status updates, etc. the BOLD effect of these areas has a high degree of consistency in different experiments. Research results have been integrated into the ACT - R cognitive system, make the ACT - R can effectively forecast the BOLD response of these regions ${ }^{[21-23]}$.

In this paper, adopt non-carry addition calculation under different emotional stimulus for experimental paradigm, using ACT-R meets fMRI to study the noncarry addition calculation under the different emotional pictures. According to the function of the eight ACT - R modules and synergy work principle, because of ACT $\mathrm{R}$ without emotion module by adjusting the length of time to indirect reaction the degree of emotional impact on cognitive computing. Experimental results based on the questionnaire survey and the behavior hypothesis model to establish the hypothesis model, model and simulate the cognitive process of the addition calculation under different emotional pictures stimulus, the experimental results verify the validity of the behavior experiment data

\section{Materials and Methods}

\subsection{Subjects}

MDD: 23 (male:15 female:8) average aged (32.76 \pm $2.48)$, average education $(10.37 \pm 1.62)$ subjects were in depressive episodes come from Anding Hospital, Capital Medical University, accord with Chinese classification and diagnostic criteria by mental disorders in version 3 (CCMD - 3), ruled out with organic mental disorder or mental active substance and the addiction caused by depression, serious body disease, severe side effects of psychotropic drugs, alcohol and substance abuse.

Control: From the same region, according to the group of the patient's gender, age, level of education, such as indicators, workers and their families in Beijing university of technology, such as student volunteers in matching selection, selection of standard is: without mental illness, neurological diseases, serious body disease history and voluntary cooperation. 23subjects (male:15 female:8), average aged (31.72 \pm 2.63$)$, average education $(9.88 \pm 1.58)$, as shown in Tab.1.

In the two groups had no age $(\mathrm{t}=0.41, \mathrm{P}=0.59)$, level of education $(\mathrm{t}=0.03, \mathrm{P}=0$. 76) had no significant differences.
Table 1 . General demographic information of MDD and control

\begin{tabular}{|l|l|l|}
\hline & Control & MDD \\
\hline Gender(male/female) & $15 / 8$ & $15 / 8$ \\
\hline age & $31.72 \pm 2.63$ & $32.76 \pm 1.48$ \\
\hline education & $9.88 \pm 1.58$ & $10.37 \pm 0.62$ \\
\hline
\end{tabular}

\subsection{Stimuli and Experimental Design}

An experimental procedure compiled and run in E prime 2.0 (Psychological Software Tools, Inc., Sharpsburg, PA, USA). The stimulate content is presented to subjects by MRI machine by vision systems, subjects may react by button box. The reaction time and accuracy is recorded automatically by program. Before formal experiment, using unified sequence of instructions and practice to train participants to ensure that the participants understand the experiment content and operation method.

Experiment tasks using the addition calculate equation under different emotional stimuli as experimental paradigm, control: addition calculate equation under positive emotional stimuli (CP), addition calculate equation under neutral emotional stimuli $(\mathrm{CNe})$, addition calculate equation under negative emotional stimuli (CN); MDD: addition calculate equation under positive emotional stimuli (MP), addition calculate equation under neutral emotional stimuli (MNe), addition calculate equation under negative emotional stimuli $(\mathrm{MN})$. Firstly present an emotional picture (2s), then present an addition calculation equation (4s) and judge the equation right or wrong. It asked participants to judge the equation as soon as possible as shown in Tab.2. Using experimental block design, the experiment contains 18 blocks, and each block contains three trials, each trial contains one emotional stimuli picture and one equation, the picture and equation alternate rendering as shown in Fig.1, Whole experiment duration 486s.

Table 2. Examples of experimental tasks

\begin{tabular}{|l|l|l|l|l|}
\hline & Task & Options & Answer \\
\hline positive & $12+14=26$ & A.right & B.wrong & A \\
\hline neutral & $13+15=38$ & A.right & B.wrong & B \\
\hline negative & $14+13=27$ & A.right & B.wrong & A \\
\hline
\end{tabular}

\subsection{MR Data Acquisition}

Scanning was performed on a $3.0 \mathrm{~T}$ MRI system (Siemens Trio Tim; Siemens Medical System, Erlangen, Germany) with a 12-channel phased array head coil. Foam paddings and headphones were used to limit head motion and reduce scanning noise. Structural images were obtained by using a three-dimensional magnetization prepared rapid acquisition gradient echo (3-D MPRAGE) sequence with the following parameters: 
repetition time $(\mathrm{TR}) /$ echo time $(\mathrm{TE})=1600 / 2.25 \mathrm{~ms}$, flip angle $=9^{\circ}, 192$ sagittal slices, voxel size $=1 \times 1 \times 1$ $\mathrm{mm} 3$. Functional data were acquired using a $\mathrm{T} 2 *$ gradient echo-planar imaging (EPI) pulse sequence $(\mathrm{TR} / \mathrm{TE}=2000 / 31 \mathrm{~ms}, 30$ axial slices, voxel size $=3.75$ $\times 3.75 \times 4.0 \mathrm{~mm} 3,0.8 \mathrm{~mm}$ inter-slice gap, $90^{\circ}$ flip angle, $64 \times 64$ matrix size in $240 \times 240 \mathrm{~mm} 2$ field of view [FOV]). This acquisition sequence generated 364 volumes for each session. The presentation of every trial was synchronized with the scanner.

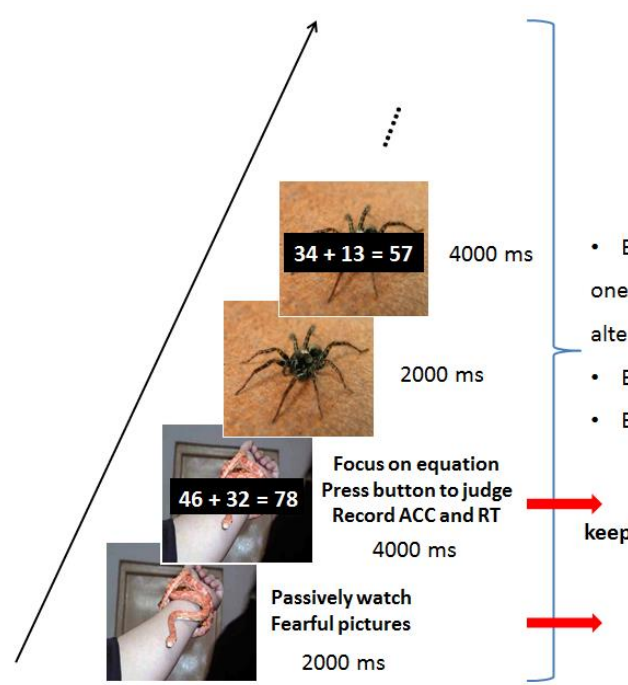

Fig.1. Paradigm of stimuli presentation one picture and one equatipaged within the two ROIs. In order to relate our alternate rendering results to previous studies, we reported the contrast - Each block containsæBStsiesiated with two simple main effects of Task (CPEach block lasts $18 \mathrm{CN}$ ) and (MP-MN), but are primarily interested in the $6 \mathrm{~s} \times 3=18 \mathrm{~s}$ activations common to both strategies $[(\mathrm{CP}-\mathrm{CN})$ and To make subjec(sMP-MN)]. The common activation map was achieved the emotion and foutfte following two steps: The map of $(\mathrm{CP}-\mathrm{CN})$ was first outputted as a mask, and then (MP- MN) was

To arouse examined within the mask. The Task by Content subjects' emotian interaction comparisons $[(\mathrm{CP}-\mathrm{CN})-(\mathrm{MP}-\mathrm{MN})]$ and $[(\mathrm{MP}-\mathrm{MN})-(\mathrm{CP}-\mathrm{CN})])$ were also executed to reveal the areas specific to each strategy. The activation reported survived a voxel-level intensity threshold of $\mathrm{p}=0.05$ corrected for false discovery rate (FDR) for the whole brain volume with a minimum cluster size of 10 contiguous voxels.

Data were analyzed using SPM8 software (http://www.fil.ion.ucl.ac.uk). The first four images for each session were discarded to allow for T1 equilibration effects. The remaining fMRI images were first corrected for within-scan acquisition time differences between slices and then realigned to the first volume to correct for inter-scan head motions (Head movement was b $2 \mathrm{~mm}$ and $\mathrm{b} 2^{\circ}$ in all cases). The structural image was coregistered to the mean functional image created from the realigned images using a linear transformation. The transformed structural images were then segmented into gray matter (GM), white matter (WM) and cerebrospinal fluid (CSF) by using a unified segmentation algorithm. The realigned functional volumes were spatially normalized to the Montreal Neurological Institute (MNI) space and re-sampled to $3 \mathrm{~mm}$ isotropic voxels using the normalization parameters estimated during unified segmentation. The registration of the functional data to the template was checked for each individual subject. Subsequently, the functional images were spatially smoothed with a Gaussian kernel of $8 \times 8 \times 8 \mathrm{~mm} 3$ full width at half maximum (FWHM) to decrease spatial noise.

\section{5 fMRI Analysis}

Only the correct response trials were included in data analysis (wrong response trials and interferential trials were not included). The epoch of interest is the duration

\subsection{ACT-R Modeling}

Having now reviewed the result of the experiment, we come to the question whether we can understand them in the framework of the ACT-R theory.

The model we constructed primarily depends on the visual module to perceive the stimuli, the manual module to respond, the retrieval module to retrieve a fact from memory, and the imaginal module to encode and update its stored representation. Communication among these modules is achieved through a procedural module. For example, the following might be a production rule for producing of the addition under neural emotional stimulus of control:

IF the goal is to solve the addition calculation problem and the problem is of the form "Number1 Number2 Number3" and "Number1 + Number2 = Number3" has been retrieved

THEN the answer of the problem is Number3.

As the fronto-parietal network plays an important role in additional calculate process, the retrieval and imaginal module are of special interest in the present study. In the following, we would illustrate the prediction of BOLD responses in the two modules. Fig.2 and Fig. 3 illustrates the sequences of activities in the four relevant buffers of ACT-R, by taking addition 
("23+35=58") under different emotional stimulus of control and addition (" $23+35=58$ ") under different emotional stimulus of MDD as examples. Each box reflects a period of time during which that buffer is active, and the vertical length of the box reflects the duration that the buffer is active. With the processes in Fig. 2 and Fig. 3 and their durations set, we will test whether the BOLD responses in the fronto-parietal regions can be predicted.

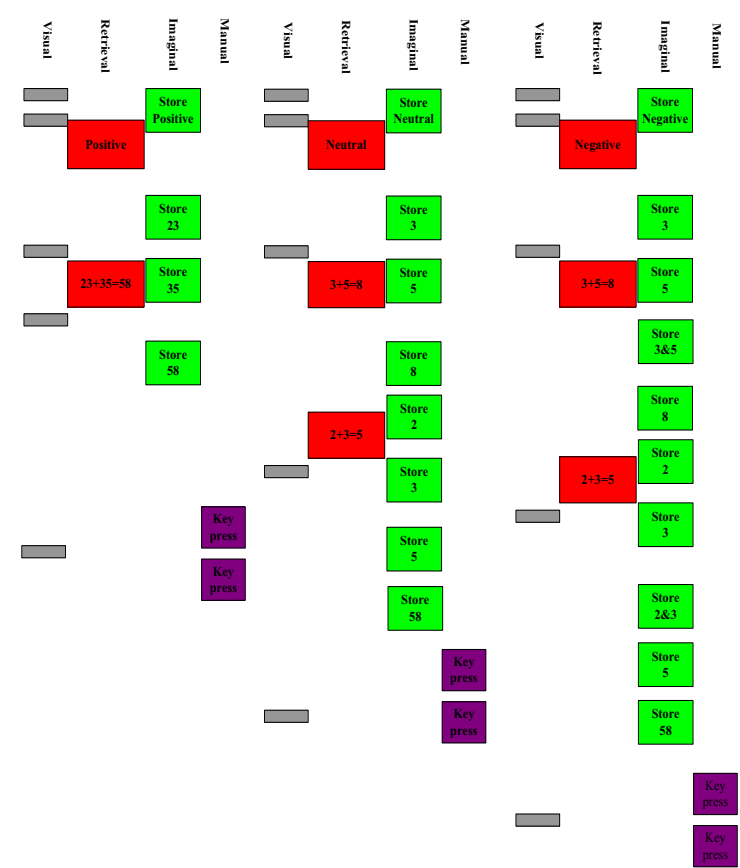

Fig.2. A schematic representation of the ACT-R model's solution to addition under different emotions of control

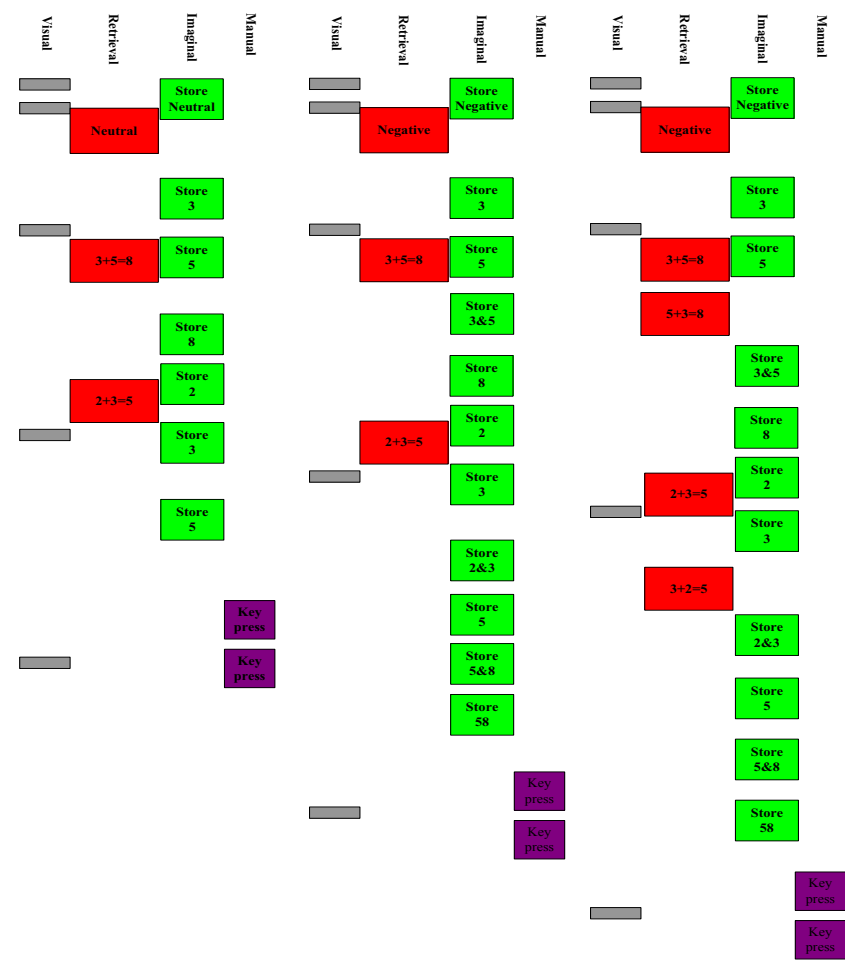

Fig.3. A schematic representation of the ACT-R model's solution to addition under different emotions of MDD

\section{Results}

\subsection{Behavioral Performances}

We carried out analyses of variance for two factors: Content (number vs. letter) and Task (induction vs. baseline) on both RT and accuracy. Behavioral scores indicated that subjects performed the task in the expected manner (Tab.3).

Table 3. Behavioral scores

\begin{tabular}{|l|l|l|l|}
\hline & \multicolumn{2}{|l|}{ RT(ms) } \\
\hline & positive & neutral & negative \\
\hline control & $1861 \pm 372.36$ & $2092 \pm 719.61$ & $2283 \pm 928.05$ \\
\hline MDD & $2348 \pm 569.42$ & $2481 \pm 983.08$ & $2704 \pm 762.42$ \\
\hline
\end{tabular}

\begin{tabular}{|l|l|l|l|}
\hline \multicolumn{3}{|l|}{ Accuracy(\%) } \\
\hline & positive & neutral & negative \\
\hline control & $96 \pm 7.34$ & $93 \pm 4.86$ & $89 \pm 11.04$ \\
\hline MDD & $91 \pm 4.15$ & $88 \pm 3.87$ & $86 \pm 5.03$ \\
\hline
\end{tabular}

\subsection{Results of ACT-R Modeling}

The fit of the predictions of the model to the RT and ACC data are presented in Fig.7. The parameters were estimated to fit the behavioral data: a factor that scaled the time to retrieve a declarative memory fact $(0.4 \mathrm{~s})$ and the time to modify the contents of the imaginal module (i.e., $0.2 \mathrm{~s}$ ). We fixed $\alpha$ to be 2 and s to be $2 \mathrm{~s}$. Once the timings of buffer actions are all set, we can predict the BOLD functions by estimating the magnitude parameter $\mathrm{m}$ from the BOLD response in each brain region ${ }^{[22-24]}$. The estimates of these parameters and measurement of the quality of the prediction are given in Tab.4 and Tab.5. Fig. 8 and Fig.9 displays percent change of BOLD response (relative to the baseline defined by the average BOLD response of the first two scans and the last two scans), along with the prediction of the ACT-R model to be presented.

Table 4. Parameters and the quality of the BOLD function prediction of control

\begin{tabular}{|l|l|l|}
\hline & Imaginal & Retrieval \\
\hline Exponent $(\alpha)$ & 1.8 & 1.8 \\
\hline Scale(s) & 1.8 & 1.8 \\
\hline Magnitude & & \\
\hline $\mathrm{M} \Gamma(\alpha+1)$ & 5.96 & 3.57 \\
\hline Correlation(r) & 96 & 93 \\
\hline
\end{tabular}

*This is a more meaningful measure because the height of the function is determined by the exponent as well as M.

Table 5. Parameters and the quality of the BOLD function prediction of MDD

\begin{tabular}{|l|l|l|}
\hline & Imaginal & Retrieval \\
\hline Exponent $(\alpha)$ & 2.1 & 2.1 \\
\hline Scale $(\mathrm{s})$ & 2.1 & 2.1 \\
\hline Magnitude & & \\
\hline $\mathrm{M} \Gamma(\alpha+1)$ & 6.24 & 4.92 \\
\hline Correlation $(\mathrm{r})$ & 97 & 94 \\
\hline
\end{tabular}




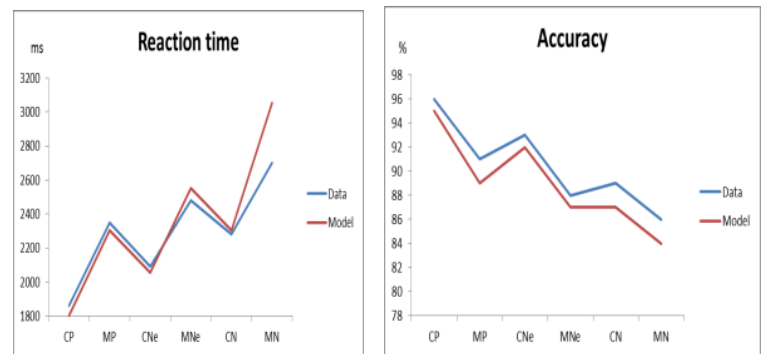

Fig.7 Data (blue lines) and Model(red lines) for addition under different emotional stimulus of control and MDD

(NOTE: C- denotes control, M- is MDD)
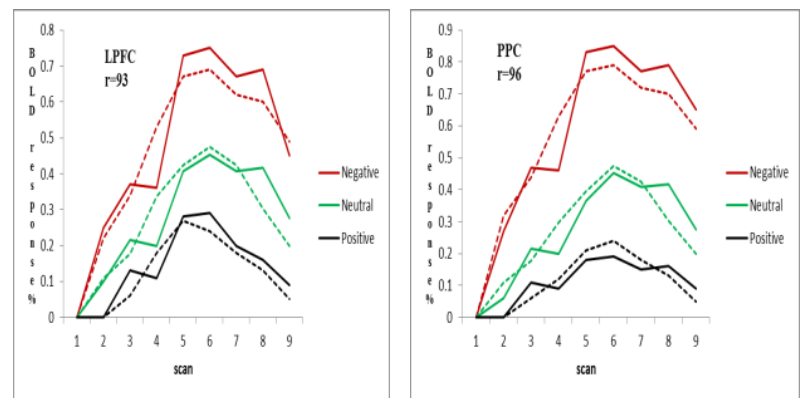

Fig.8 BOLD functions obtained for the prefrontal and parietal regions for addition under different emotional stimulus of control

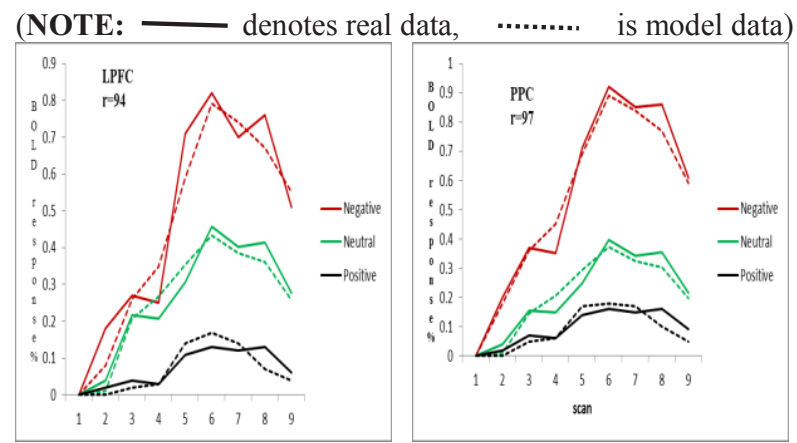

Fig.9 BOLD functions obtained for the prefrontal and parietal regions for addition under different emotional stimulus of MDD

(NOTE: - denotes real data, is model data)

\section{Conclusion}

MDD emotional active areas and cognitive function areas may have damage ${ }^{[9]}$. So reaction time of control for addition in the same emotional stimuli is shorter than MDD, at the same have higher accuracy; it has a retention phenomenon due to the negative sentiment ${ }^{[13-15]}$. So in the same group, reaction time for addition under positive emotional stimuli is shorter than negative emotions, and the accuracy is higher. The ACT-R model and simulation results for addition under different emotional stimuli verify the hypothesis.

\section{References}

[1] Neugebauer R. Mind matters:the importance of mental disorders in public health' $\mathrm{s} 21^{\text {st }}$ century mission $[\mathrm{J}]$. Am J Pub Health, 1999, 89: 1309-1311.
[2] Mayberg H S, Lozano A M, VoonV, et al. Deep brain stimulation for treatment-resistant depression [J]. Neuron, 2005, 45: 651-660.

[3] Baref B R. Introduction to functional magnetic resonance imaging:principles \& techniques[M]. London: Cambridge University Press, 2002:272.

[4] Zhang D, Raichle M E. Disease and the brain's dark energy [J].Nat. Rev. Neurol., 2010, 6: 15-28.

[5] Bower G H, Forgas J P. Affect, memory, and social cognition. In: Eich E, Kihlstrom J F, Bower G H, et al., eds. Cognition and Emotion. New York: Oxford University Press, 2000. 87-168.

[6] Langeslag S J E, Franken I H A, van Strien J W. Dissociating love-related attention from task-related attention: An event-related potential oddball study [J]. Neurosci Lett, 2008, 431: 236-240.

[7] Fox M D, Raichle M E. Spontaneous fluctuations in brain activity observed with functional magnetic resonance imaging [J]. Nat.Rev. Neurosci., 2007, 8: 700-711.

[8] Raichle M E.The brain's dark energy [J]. Sci. Am., 2010, 302: 44-49.

[9] Sharp S C, Hellings J A. Efficacy and safety of selective serotonin reuptake inhibitors in the treatment of depression in children and adolescents: practitioner review [J]. Clin Drug Investig, 2006, 26(5): 247-255.

[10] Murray C J L, Lopez A D. Alternative projections of mortality and disability by cause 1990-2020: Global Burden of Disease Study [J]. Lancet, 1997, 349: 14981504.

[11] Pessoa L. On the relationship between cognition and emotion [J]. Nat Rev Neurosci, 2008, 9: 148-158.

[12] Lyons W. The philosophy of cognition and emotion. In: Dalgleish T, Power M, eds. Handbook of Cognition and Emotion. Chichester: Wiley, 1999. 21-44.

[13] Ochsner K N, Phelps E. Emerging perspectives on emotion-cognition interactions [J]. Trends Cogn Sci, 2007, 11: 317-318.

[14] Dolan R. Emotion, cognition, and behavior [J]. Science, 2002, 298: 1191-1194.

[15] Barrett L F. Emotions as natural kinds? [J]. Perspect Psychol Sci, 2006, 1: 28-58.

[16] Pessoa L. How do emotion and motivation direct executive control? [J]. Trends Cogn Sci, 2009, 13: 160166.

[17] Barrett L F, Mesquita B, Ochsner K N, et al. The experience of emotion [J]. Annu Rev Psychol, 2007, 58: 373-403.

[18] Izard C E. Emotion Theory and research: Highlights, unanswered questions, and emerging issues [J]. Annu Rev Psychol, 2009, 60: 1-25.

[19] Ekman P. Handbook of Cognition and Emotion [M]. Chichester: Wiley, 1999.

[20] Mehrabian A. Framework for a comprehensive description and measurement of emotional states [J]. Genet Soc Gen Psychol Monogr, 1995, 3: 339-361.

[21] Martino B D, Kumaran D, Seymour B, et al. Frames, biases, and rational decision-making in the human brain [J]. Science, 2006, 313:684-687.

[22] Irrational Decisions Driven By Emotions, ScienceDaily. Retrieved June 29, 2009, from http://www.sciencedaily. com/releases/ 2006/08/060803171138.htm.

[23] Clore G L, Storbeck J. Affect as information about liking, efficacy, and importance. In: Forgas J, ed. Affect in Social Thinking and Behavior [J]. New York and Hove: Psychology Press, 2006.104: 123-142.

[24] Buchanan T W. Retrieval of emotional memories [J]. Psychol Bull, 2007, 133: 761-779 